\title{
Distal nebulin myopathy
}

INSERM

\section{Source}

INSERM. (1999). Orphanet: an online rare disease and orphan drug data base. Distal nebulin myopathy. ORPHA:399103

Distal nebulin myopathy is a rare, slowly progressive, autosomal recessive distal myopathy characterized by early onset of predominantly distal muscle weakness and atrophy affecting lower leg extensor muscles, finger extensors and neck flexors. Muscle histology does not always show nemaline rods. 